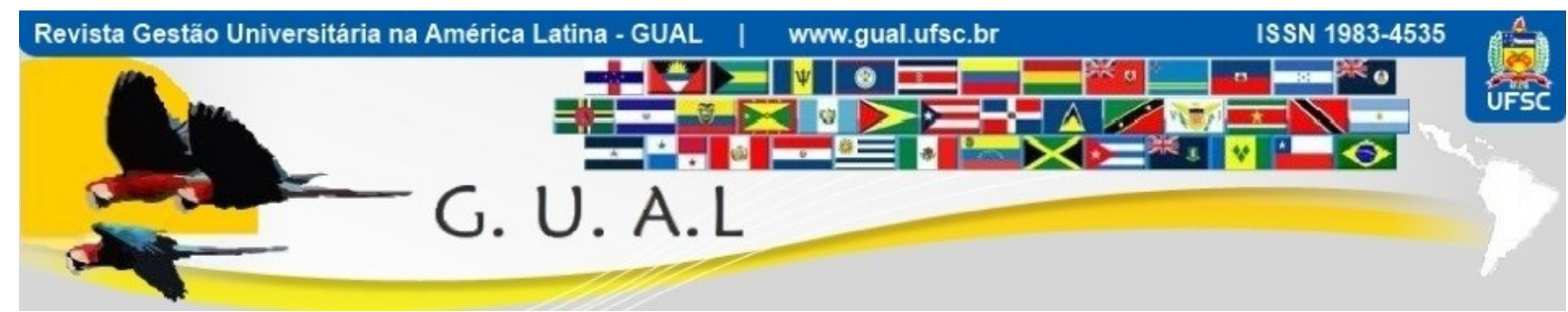

DOI: http://dx.doi.org/10.5007/1983-4535.2018v11n4p127

\title{
APLICAÇÃO DE MODELO DE MENSURAÇÃO DA SATISFAÇÃO DOS USUÁRIOS DE UM SISTEMA DE INFORMAÇÃO ACADÊMICO: ESTUDO DE CASO NA UNIVERSIDADE FEDERAL DO RIO GRANDE DO NORTE
}

\begin{abstract}
APPLICATION OF A MEASUREMENT MODEL FOR THE SATISFACTION OF USERS OF AN ACADEMIC INFORMATION SYSTEM: A CASE STUDY AT THE FEDERAL UNIVERSITY OF RIO GRANDE DO NORTE
\end{abstract}

Fernanda Cristina Barbosa Pereira Queiroz, Pós-Doutora

Universidade Federal do Rio Grande do Norte - UFRN fernandacbpereira@gmail.com

Ana Clara Cachina, Mestre Universidade Federal do Rio Grande do Norte - UFRN anaclaracachina@hotmail.com

Luana Pinheiro, Mestre Universidade Federal do Rio Grande do Norte - UFRN luanapinheiro@hotmail.com

Anna Camila Lima e Silva, Mestre Universidade Federal do Rio Grande do Norte - UFRN anna.camila@hotmail.com

Eduardo Lopes Marques, Doutor Universidade Federal de Viçosa - UFV eddumarques1@gmail.com

Recebido em 24/novembro/2017 Aprovado em 06/setembro/2018
Maria do Carmo Duarte Freitas, Doutora Universidade Federal do Paraná - UFPR carmemk2@gmail.com

Sistema de Avaliação: Double Blind Review 


\title{
RESUMO
}

O sistema de informação proporciona um meio de comunicação e de troca de informações entre os diferentes públicos, tornando a geração e disseminação de informações um processo ágil e eficaz. As Universidades Federais do Brasil investem na informatização dos processos acadêmicos e administrativos buscando excelência nas atividades de gestão e sobretudo nas atividades de ensino, pesquisa e extensão. Este artigo tem como objetivo aplicar o modelo de avaliação da satisfação, desenvolvido por Ainin, Bahri e Ahmad (2012), nos usuários do sistema acadêmico de uma Universidade. A metodologia empregada consistiu no envio de um questionário eletrônico para os alunos da Universidade Federal do Rio Grande do Norte (UFRN) e coleta de 1658 respostas que foram analisadas utilizando-se a modelagem de equações estruturais para identificar os fatores que contribuem com o processo investigado. $\mathrm{O}$ estudo observou todos os critérios estatísticos para validação, além de apresentar um bom nível de ajuste, mostrando-se estatisticamente adequado. $\mathrm{O}$ fator utilidade percebida teve maior impacto na determinação da satisfação dos usuários, seguido pela Qualidade do Serviço, Qualidade da Informação e Qualidade do Sistema.

Palavras-chave: Sistema de informação acadêmico. Modelagem de Equações Estruturais. Satisfação dos usuários.

\begin{abstract}
The information system provides the generation and dissemination of information an agile and effective process among different audiences. The Federal Universities of Brazil invest in the computerization of the processes seeking excellence in the activities. This article aims to apply the Satisfaction Evaluation model, developed by Ainin, Bahri and Ahmad (2012), in the users of the academic system of a University. The methodology used consisted of sending an electronic questionnaire to the students of the Federal University of Rio Grande do Norte and collecting 1658 responses that were analyzed using the Structural Equations Modeling. The study observed all the statistical criteria for validation, in addition to presenting a good level of adjustment, showing to be statistically adequate. The Perceived Utility factor had a greater impact on the determination of User Satisfaction, followed by Quality of Service, Quality of Information and System Quality.
\end{abstract}

Keywords: Academic information system. Structural equations modeling. User satisfaction. 


\section{INTRODUÇÃO}

O presente estudo discute a necessidade de avaliar a satisfação dos usuários dos sistemas acadêmicos das Instituições de Ensino Superior (IES). Com o desenvolvimento tecnológico houve um aumento no acesso às informações, com o intuito de aprimorar a eficiência e a qualidade, em distintas atividades econômicas, dentre elas empresarial, hospitalar e educacional (CLAYTON et al., 1992). O acesso à informação é necessário para o desenvolvimento de qualquer atividade, no entanto o cotidiano dos novos negócios exige que esse processo seja agilizado, para evitar o fluxo de informações incorretas ou desnecessárias.

A difusão da Tecnologia da Informação (TI) beneficia as organizações com rapidez, agilidade e flexibilidade para receber, processar e transformar dados em informação com eficiência e eficácia. O desenvolvimento da TI trouxe oportunidades para as empresas se reestruturarem, além de tornar possível a integração de sistemas para atender aos processos de negócio e suportar o fluxo de informação associado.

$\mathrm{O}$ avanço da tecnologia e a extensão da complexidade do fluxo informacional favoreceram para o surgimento dos Sistema de Informações Gerenciais Integrados. A demanda crescente pelo uso de informação com qualidade, considerando a dimensão tempo, conteúdo e forma, transcenderam o universo das organizações empresariais para alcançar novos patamares de usabilidade como o do universo acadêmico (SANTOS, 2006).

As IES precisam de sistemas que as auxiliem no gerenciamento de informações necessárias à gestão e às atividades acadêmicas de ensino, pesquisa e extensão. Estas instituições utilizam os Sistemas de Informações Acadêmicos (SIAs), que são a base para uma gestão moderna nas IES (SILVA, 2012).

Sendo assim, nos âmbitos empresarial e acadêmico houve uma difusão dos Sistemas Integrados de Gestão (SIG), que têm como objetivo integrar toda a gestão da empresa com a obtenção de informações em tempo real, agilizando assim o processo de tomada de decisão (CLAYTON et al., 1992). Na esfera acadêmica, existem informações que são utilizadas na realização de serviços e efetivação de processos dos servidores, docentes e discentes.

É neste ponto que se formula a questão que orienta esta pesquisa: Quais os fatores determinantes da satisfação dos usuários de um sistema de informação acadêmico? Diante destas considerações, o objetivo da presente pesquisa é aplicar um modelo de determinação da satisfação dos usuários de um sistema de informação e analisar os dados por meio da 
Modelagem de Equações Estruturais (MEE), com vistas a mensurar e explicar os construtos que agem sobre a satisfação, para entender os fatores influenciadores na satisfação dos discentes.

Esta pesquisa se justifica por permitir avaliar a satisfação dos alunos na Universidade Federal do Rio Grande do Norte em relação ao uso dos SIAs, colaborando para a melhoria contínua dos sistemas de informações gerenciais, por meio da sua perspectiva em relação ao que é ofertado.

Este artigo encontra-se estruturada em cinco capítulos unidos, com o intuito de gerar maior clareza para as discussões abordadas e fornecer subsídios para responder os objetivos de pesquisa.

O capítulo 1 - Introdução - delimita os temas a serem abordados no artigo, abordando a necessidade de avaliar a satisfação dos usuários dos sistemas acadêmicos das Instituições de Ensino Superior (IES

O capítulo 2 - Fundamentação teórica - aborda os sistemas de informações acadêmicas, satisfação do usuário e os modelos de satisfação.

O capítulo 3 - Método da pesquisa - destaca os procedimentos metodológicos utilizados, desde a seleção das referencias utilizadas no capítulo anterior até a coleta e tratamento dos dados. São definidas as hipóteses de pesquisa, a formulação do modelo teórico e as variáveis a serem investigadas, os critérios de escolha dos métodos, instrumento de coleta de dados, definição da população e amostra, e como será o processo de validação do modelo. Outro ponto tratado foi a Modelagem de Equações Estruturais .

O capítulo 4- Resultados e Discussões - traz a aplicação da Modelagem de Equações Estruturais em dados oriundos do questionário aplicados aos usuários do SIGAA, avaliando o perfil dos usuários do sistema da UFRN e os determinantes da satisfação.

O capítulo 5 - Considerações Finais - encerra este trabalho apresentando os principais resultados da pesquisa, respondendo aos objetivos propostos e as hipóteses levantadas.

\section{REFERENCIAL TEÓRICO}

Este capítulo aborda resgates teóricos indispensáveis em face dos objetivos propostos neste artigo. A fundamentação teórica, proposta contempla a discussão dos temas como: Sistemas de informações acadêmicas e satisfação do usuário. E neste capítulo, discussões de importantes autores de cada um dos temas são apresentadas e debatidas. 


\subsection{SISTEMA DE INFORMAÇÕES ACADÊMICAS}

No início da década de 1990 o Sistema Integrado de Gestão (SIG) começou a ser adotado nas empresas brasileiras (MATTOS, 1999). Nos últimos anos, o SIG está em evidência no mercado brasileiro, sendo destacado como ferramenta para o suporte das operações e do gerenciamento das organizações, dentre elas, as IES. O uso de ferramentas tecnológicas permite o acesso a informações de forma mais ágil possibilitando que as decisões tomadas sejam racionais e colaborando com comunicação entre os alunos e professores.

A necessidade de disponibilizar as funcionalidades de controle relacionadas a dados cadastrais dos discentes, dos docentes, do curso e de acesso a informações e a ações de disciplinas, de requisitos, de ofertas de turmas, de matrícula de discentes, de lançamento de notas pelos docentes, de histórico escolar, e outras utilidades auxiliam os gestores de instituições de ensino (CARVALHO et al., 2002).

Portanto, as novas tecnologias devem apresentar precisão em sua utilização, devido à quantidade de informações geradas nas universidades e que, conseqüentemente, precisam ser acessadas, coletadas, filtradas, processadas e examinadas (SENGER; BRITO, 2005). De acordo com Reis et al. (2012), a adoção de novas tecnologias tem sido um dos maiores desafios para as IES.

Neste contexto, o Sistema de Informação Acadêmica permite que o aluno tenha maior agilidade ao interagir com a instituição, com o curso e com os professores, acessando via internet, os dados e informações necessários. Desse modo, as IES têm encontrado maior facilidade em gerenciar recursos de informação e apoio administrativo aos serviços de educação, que foram simplificados e individualizados de forma segura, consistente e personalizada.

Segundo Lima (2006), a utilização desses novos instrumentos na gestão acadêmica, aprova um máximo de controle na vida acadêmica de alunos. Os administradores das instituições de ensino, pelo novo formato de informação, podem acessar uma grande quantidade unificada e concentrada, e simultaneamente gerir respostas (LEE et al., 2009).

Para Silva (2012), o SIA é uma ferramenta de controle administrativo nas instituições de ensino, tanto de processos administrativos quanto gerenciais. Ainda assim, segundo o autor não alcança as reais necessidades dos docentes e discentes envolvidos. Assim, é preciso desenvolver e aperfeiçoar as práticas e instrumentos de gestão universitária. Coletando e 
sistematizando informações de forma confiável, proporcionando aos gestores ações mais efetiva e focada (PEIXOTO; BASTOS, 2013).

\subsection{A SATISFAÇÃO DO USUÁRIO}

A satisfação é um tema abordado nos estudos sobre o comportamento do consumidor, e grande parte dos teóricos desta área defendem a correlação direta entre os índices de satisfação e a lealdade do cliente (SILVA, 2015). Ao concentrar seus estudos em satisfação em TIC's, Doll e Torkzadeh (1988) relatam que a satisfação do usuário é definida como o parecer do usuário sobre uma aplicação informática específica ou sistema de informação disponível para eles que atenda às suas necessidades de informação. Igualmente, Donget et al. (2014) trazem uma definição de satisfação do usuário especialmente para TIC, onde se interessa pelas atitudes dos usuários a sistemas de computadores no contexto da sua ambientes.

Para Oliver (1980) a satisfação é uma resposta afetiva geral a uma discrepância percebida entre as expectativas anteriores e desempenho percebido após o consumo. Segundo DeLone e Mclean (1992), a satisfação do usuário é uma das formas mais importantes de mensuração do sucesso de um SI. Para Ainin, Bahri e Ahmad (2012) a satisfação do usuário refere-se à interação bem-sucedida entre o próprio sistema de informação e seus usuários. $\mathrm{O}$ usuário identifica a satisfação em ser atendido de acordo com o que espera do sistema.

É importante identificar o que os clientes valorizam em um conjunto específico de serviços, com a intenção de maximizar a satisfação do usuário e retê-lo. Isso introduz um novo requisito: para medir a satisfação do cliente como fator de contínua melhoria dos negócios (BOURNARIS et al., 2013). Portanto, a satisfação de usuários que utilizam sistema de informação depende da análise de critérios que influenciam positivamente ou negativamente, necessitando de modelos para a análise.

\subsubsection{Modelo de satisfação}

A satisfação dos usuários de sistemas pode ser mensurada por meio de modelos propostos por autores como DeLone e McLean $(1992,2003)$ que avaliaram que a qualidade do serviço, a qualidade do sistema e a qualidade de informação, conjuntamente ou em separado, influenciam a satisfação do usuário e o uso do sistema, que por sua vez influencia o sucesso 
do Sistema de Informação - SI. Os autores propuseram que um sistema de alta qualidade está relacionado com mais uso, mais satisfação do usuário e os benefícios resultantes.

Ainin, Bahri e Ahmad (2012) apresentaram um modelo, desenvolvido na Malásia, para avaliar a satisfação dos estudantes, adaptado das pesquisas de Wixon e Todd (2005) que buscavam integrar as percepções de satisfação e tecnologia para se avaliar os sistemas de informação e também nas pesquisas de Ong et al (2009) que avaliaram a satisfação dos usuários dos sistemas de respostas automáticas a questões (QAS).

\section{METOLOGIA DA PESQUISA}

\subsection{HIPÓTESES DA PESQUISA}

O presente estudo adaptou o modelo elaborado por Ainin, Bahri e Admad (2012) de acordo com a realidade da população estudada. O modelo proposto por Ainin, Bahri e Ahmad (2012) foi elaborado para mensuração da satisfação do usuário considerando quatro constructos: qualidade do sistema, qualidade da informação, qualidade do serviço e utilidade percebida, apresentados em síntese na Figura 1.

Figura 1 Modelo de Satisfação do Usuário

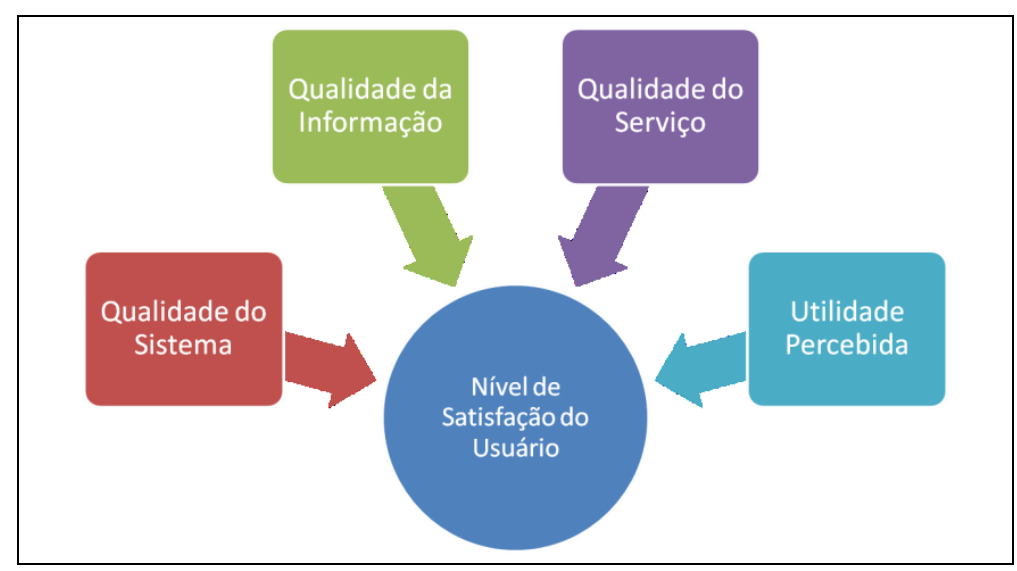

Fonte: Adaptado de Ainin, Bahri e Ahmad (2012)

O modelo considera a variável latente, ou constructo, qualidade do sistema como as características inerentes ao sistema como erros, consistência de navegação, facilidade de uso, entre outros. De acordo com Roldán e Leal (2006), qualidade do sistema refere-se às características desejadas dos SI, que produzem a informação e estão relacionadas com a qualidade do output do SI. Assim, a primeira hipótese do trabalho pode ser descrita em H1. 
- $\quad$ "H1 - A qualidade do sistema influencia positivamente o nível de satisfação do usuário".

A qualidade da informação refere-se à informação fornecida pelo sistema e à questões de integridade, veracidade, pontualidade e disseminação. Neste aspecto, é importante assegurar a segurança da informação, para que o usuário sinta-se seguro ao utilizar e confiar em informações fornecidas pelo SI. Para Lee e Kozar (2006), a qualidade da informação que o sistema produz e fornece favorece para o sucesso do SI, uma vez que as informações devem ser relevantes, atualizadas e de fácil compreensão. Então, a segunda hipótese a ser testada na pesquisa é $\mathrm{H} 2$.

- "H2 - A qualidade da informação influencia positivamente o nível de satisfação do usuário".

A qualidade do serviço se refere ao que é percebido pelo usuário frente ao que era esperado. De acordo com Alawneh, Al-Refai e Batiha (2013), a satisfação está relacionada com a qualidade do serviço que é uma dimensão comportamental criada pela experiência, bem como uma dimensão mental. Portanto, a terceira hipótese nesta pesquisa é descrita em H3.

- $\quad$ "H3 - A qualidade do serviço influencia positivamente o nível de satisfação do usuário".

A utilidade percebida é definida como o grau em que uma pessoa considera que a utilização de um sistema particular aumentaria seu desempenho de tarefas (Davis, 1989) e, eventualmente, seu próprio nível de satisfação (Ainin, Bahri e Ahmad, 2012). Por fim, a quarta hipótese deste trabalho é H4.

- "H4 - A utilidade percebida influencia positivamente o nível de satisfação do usuário".

Os dados empíricos para este estudo foram coletados utilizando um questionário, composto por 30 perguntas fechadas estruturadas em uma escala Likert de sete pontos, variando de (1) discordo fortemente a (7) concordo fortemente.

Foram utilizados 4 constructos ou fatores ou variáveis latentes que não podem ser medidas diretamente em função da complexidade - Qualidade do Sistema, Qualidade da 
informação, Qualidade do Serviço e Utilidade percebida - que são formadas por variáveis observadas ou manifestas obtidas pelo instrumento de coleta de dados.

$\mathrm{Na}$ Tabela 1, são mostradas as variáveis que formam o constructo Qualidade do Sistema - QS.

Tabela 1 Descrição das variáveis do constructo Qualidade do Sistema

\begin{tabular}{|ll|}
\hline QS1 & $\begin{array}{l}\text { O processo de registro é simples } \\
\text { As instruções sobre como usar o sistema estão } \\
\text { disponiveis diretamente }\end{array}$ \\
QS2 & $\begin{array}{l}\text { A informação é fornecida por meio de perguntas mais } \\
\text { frequentes e respostas } \\
\text { Informações exigidas são encontradas com número } \\
\text { minimo de cliques }\end{array}$ \\
QS4 & $\begin{array}{l}\text { Uma barra de nagevação está disponivel em todas as } \\
\text { páginas }\end{array}$ \\
QS5 & $\begin{array}{l}\text { A navegação é consistente e padronizada } \\
\text { Percorrendo as páginas e o texto são mantidos a um } \\
\text { tempo minimo }\end{array}$ \\
QS7 & $\begin{array}{l}\text { É fácil de se recuperar os erros que eu faço quando se } \\
\text { utiliza o sistema }\end{array}$ \\
QS8 &
\end{tabular}

Fonte: Elaboração Própria (2017)

A Tabela 2 apresenta a descrição das variáveis da Qualidade da Informação - QI.

Tabela 2 Descrição das variáveis do constructo Qualidade da Informação

\begin{tabular}{|ll|}
\hline QI1 & $\begin{array}{l}\text { As informações fornecidas no sistema são completas } \\
\text { As informações fornecidas no sistema são fáceis de } \\
\text { QI2 }\end{array}$ \\
entender \\
As informações fornecidas no sistema são \\
personalizadas \\
Q14 & $\begin{array}{l}\text { As informações no sistema são abrangentes } \\
\text { (compreensivas) }\end{array}$ \\
QI5 & $\begin{array}{l}\text { As informações no sistema são seguras } \\
\text { Termos e condições de seu pedido de empréstimo são } \\
\text { acessiveis }\end{array}$ \\
\hline
\end{tabular}

Fonte: Elaboração Própria (2017) 
Na Tabela 3 são descritas as variáveis da Qualidade de Serviço - QO.

Tabela 3 Descrição das variáveis da Qualidade de Serviço

\begin{tabular}{|ll|}
\hline \hline QO1 & $\begin{array}{l}\text { O sistema fornece a confirmação de aceitação } \\
\text { imediatamente } \\
\text { O sistema oferece visualização de informações inseridas } \\
\text { antes da submissão }\end{array}$ \\
QO3 & $\begin{array}{l}\text { A central de contato fornecer serviços de alerta/ } \\
\text { imediatos aos usuários }\end{array}$ \\
QO4 & $\begin{array}{l}\text { A central de contato tem o conhecimento para fazer seu } \\
\text { trabalho com eficiência e eficácia }\end{array}$ \\
QO5 & $\begin{array}{l}\text { Sistema está disponivel } 24 \mathrm{~h}, 7 \text { dias por semana } \\
\text { O número de contato e link de e-mail da central de }\end{array}$ \\
QO7 & $\begin{array}{l}\text { contato estão disponiveis } \\
\text { Consultas ou queixas são resolvidas com } 24 \mathrm{~h}\end{array}$ \\
QO8 & $\begin{array}{l}\text { O sistema proporciona segurança de dados, ou seja, } \\
\text { senha acessar informações detalhadas }\end{array}$ \\
\hline
\end{tabular}

Fonte: Elaboração Própria (2017)

A descrição das variáveis que formam a variável latente Utilidade Percebida - UP é apresentada na Tabela 4.

Tabela 4 Descrição das variáveis do constructo Utilidade Percebida

\begin{tabular}{|c|c|}
\hline UP1 & Acho que é fácil acessar este sistema \\
\hline & A quantidade de informações exibidas na tela é \\
\hline UP2 & adequada \\
\hline UP3 & A seqüência de obtenção e operação feita é clara \\
\hline UP4 & O layout das páginas faz (torna) tarefas mais fáceis \\
\hline UP5 & O sistema facilita a minha vida \\
\hline UP6 & $\begin{array}{l}\text { A taxa (medida) em que a informação foi exibida foi } \\
\text { rápida o suficiente }\end{array}$ \\
\hline UP7 & $\begin{array}{l}\text { A descrição / explicação no site para realizar minha } \\
\text { tarefa é adequada }\end{array}$ \\
\hline
\end{tabular}

Fonte: Elaboração Própria (2017)

Por fim, a variável latente endógena (dependente) Satisfação do Usuário - SATIS foi utilizada para avaliar a satisfação geral com o sistema. $O$ instrumento de coleta de dados foi enviado pela Universidade por meio de e-mail para todos os alunos matriculados. Ao final da coleta de dados, 1658 respostas válidas foram utilizadas para a realização da Modelagem de Equações Estruturais, por meio do software SmartPLS 3.2 (RINGLE et al., 2015). Para testar as quatro hipóteses apresentadas nesta pesquisa, será utilizada a Modelagem de Equações Estruturais (MEE) como método de análise de dados, conforme apresentado na seção seguinte. 


\subsection{MODELAGEM DE EQUAÇÕES ESTRUTURAIS}

A Modelagem de Equações Estruturais (MEE) é uma ferramenta estatística para modelar relações entre múltiplas variáveis. A MEE pode ser considerada uma técnica estatística de segunda geração que utiliza vários tipos de modelos para descrever relações causais entre variáveis observadas e não observadas medidas por meio de indicadores (HAIR et al, 2012; RIGDON, 2016).

As relações propostas são traduzidas em uma série de equações estruturais (semelhantes às equações de regressão) para cada variável dependente (HAIR et al., 2005). A aplicação da MEE permite ao pesquisador a obtenção de dois resultados fundamentais: a estimativa da magnitude dos efeitos entre as variáveis, podendo identificar os que possuem maior significância; e a possibilidade de testar o modelo de acordo com os dados coletados.

De forma oposta aos modelos usuais de Análise Fatorial e Regressão Linear, a MEE baseia-se num quadro teórico estabelecido a priori. Assim a hipótese nula especifica o modelo que o investigador crê ser válido, e os dados servem para demonstrar que o modelo teórico explica convenientemente (ou aproximadamente) as relações observadas entre as variáveis medidas. Outra característica que difere a AEE é que ao contrário da Regressão, o modelo pode apresentar mais de uma variável dependente (HAIR et al, 2014).

Existem duas técnicas de análise na modelagem de equações estruturais: a baseada em covariância (CB-SEM) e a de mínimos quadrados parciais (PLS-SEM). A técnica CB-SEM envolve um processo de máxima verossimilhança, onde o objetivo é o de minimizar a diferença entre as matrizes de covariância observadas e esperadas. A abordagem PLS -SEM, por outro lado, se concentra em maximizar a variância explicada (prevista) das variáveis dependentes, isto é, minimizar a variância não explicada (HAIR et al, 2012; SARSTED et al, 2016).

Como elementos do modelo teórico, as variáveis conceituais representam grandes ideias ou pensamentos sobre conceitos abstratos que os investigadores estabelecem e propõem a medição na pesquisa (por exemplo, a satisfação do usuário). Essas são usualmente classificadas entre variáveis manifestas e variáveis latentes. As variáveis manifestas são medidas, manipuladas ou observadas diretamente; enquanto as variáveis latentes, também chamadas de fatores ou constructos, são não diretamente observáveis ou mensuráveis, sendo medidas por meio de variáveis manifestas. 


\section{ANÁLISE DOS RESULTADOS}

\subsection{SISTEMA DE INFORMAÇÃO ACADÊMICA DA UFRN}

Os sistemas de informação surgiram na UFRN, na década de 70, através da contratação de softwares de outras empresas (BARROCA FILHO; AQUINO JÚNIOR, 2016). Cada área da Universidade escolhia a empresa que se adequava melhor às suas necessidades e os modelos que desejava seguir. Como consequência, não havia fluxo de informação entre os setores e estes se tornaram remotos.

Em 2000, foi criada a Superintendência de Informática da UFRN com o intuito de interligar esses módulos operacionais, criando uma só base e transmitindo as informações necessárias de um sistema para outro. Então, surgiu os três sistemas base da Universidade: Sistema integrado de Gestão de Atividades Acadêmicas - SIGAA; Sistema Integrado de Patrimônio, Administração e Contratos - SIPAC; e Sistema Integrado de Gestão de Recursos Humanos - SIGRH (BARROCA FILHO; AQUINO JÚNIOR, 2016).

Atualmente, a instituição conta com mais cinco bases de sistemas: Sistema Integrado de Gestão de Planejamento e de Projetos - SIGPP; Sistema Integrado de Gestão Eletrônica de Documentos - SIGED; Sistema Integrado de Gestão de Eleições - SIGEleição; Sistema Integrado de Gestão de Eventos - SIGEventos; e Sistema de Administração dos Sistemas SIGAdmin. São dividas principalmente em duas grandes áreas: área administrativa e área acadêmica.

O sistema SIGAA objetiva trazer mais comodidade e facilidade aos usuários da universidade, evitando a perca com deslocamento até o local, facilitar a comunicação entre departamentos e usuários-departamentos. Representa digitalmente toda a informação relacionada com o funcionamento dos cursos de graduação e pós-graduação da universidade, fornece ferramentas para exploração e navegação no espaço de informação acadêmica, e promover a gestão acadêmica assistida por computador com recurso a equipamentos de computação móvel e distribuída.

Queiroz et al (2012) identificaram que o SIGAA contribui para a melhor interação aluno e professor, por um lado auxilia na aprendizagem do aluno ao fornecer um contato mais fácil e rápido com os professores por meio de suas ferramentas e por outro lado, permite ao professor uma forma rápida e fácil de disseminar materiais e arquivos que podem ajudar aos aluno no processo de aprendizagem. 


\subsection{PERFIL DOS ALUNOS USUÁRIOS DO SIGAA/UFRN}

Os usuários do SIGAA que responderam à pesquisa são jovens, a maioria com menos de 25 anos, matriculado nos diferentes cursos e áreas do conhecimento. A amostra obteve resposta de 862 alunos do sexo masculino (52\%) e 796 alunos do sexo feminino (48\%). A maior parte dos alunos (53\%) está na Universidade a menos de dois anos como pode ser visto no quadro 1 a seguir

Quadro 1 Características dos respondentes

\begin{tabular}{|c|c|c|c|}
\hline Variável & Detalhamento & Frequência & $\%$ \\
\hline \multirow{4}{*}{ Idade } & Menos de 21 anos & 551 & $33 \%$ \\
\hline & $22-25$ anos & 418 & $25 \%$ \\
\hline & 26-29 anos & 264 & $16 \%$ \\
\hline & Mais de 30 anos & 425 & $26 \%$ \\
\hline \multirow[t]{2}{*}{ Sexo } & Masculino & 862 & $52 \%$ \\
\hline & Feminino & 796 & $48 \%$ \\
\hline \multirow{5}{*}{ Área } & Saúde & 309 & $19 \%$ \\
\hline & Ciências Exatas & 492 & $30 \%$ \\
\hline & Ciências Humanas & 520 & $31 \%$ \\
\hline & Tecnologias & 201 & $12 \%$ \\
\hline & $\begin{array}{l}\text { Ciências } \\
\text { Aplicadas }\end{array}$ & 132 & $8 \%$ \\
\hline \multirow{7}{*}{ Tempo na Universidade } & Não respondeu & 4 & $0 \%$ \\
\hline & 1 ano ou menos & 517 & $31 \%$ \\
\hline & 2 anos & 358 & $22 \%$ \\
\hline & 3 anos & 216 & $13 \%$ \\
\hline & 4 anos & 195 & $12 \%$ \\
\hline & 5 anos & 92 & $6 \%$ \\
\hline & Mais de 5 anos & 280 & $17 \%$ \\
\hline \multirow{3}{*}{ Frequência de uso } & Diariamente & 825 & $50 \%$ \\
\hline & Semanalmente & 740 & $45 \%$ \\
\hline & Mensalmente & 93 & $6 \%$ \\
\hline TOTAL GERAL & & 1658 & $100 \%$ \\
\hline
\end{tabular}

Fonte: Elaboração Própria (2017) 


\subsection{DETERMINANTES DA SATISFAÇÃO}

\subsubsection{Resultados do Modelo de Mensuração}

O primeiro aspecto observado foi com relação à curtose e à assimetria. Os indicadores foram consistentes, visto que as distribuições das variáveis observadas não foram grandes (HAIR, 2014). A seguir, na análise das cargas fatoriais foi estabelecido que as cargas exteriores das construções reflexivas deveriam estar acima do limite de valor de 0,708 de carregamento. A variável QS5 foi excluída do modelo por apresentar carga fatorial inferior a 0,708 e modelo resultante, com todas as outras variáveis restantes, apresentou os valores de variância média extraída (AVE) e confiabilidade, medidas por meio do alfa de Cronbach e confiabilidade composta e Rho, apresentados na tabela 5. Todos os valores encontrados para as variáveis analisadas foram superiores aos valores mínimos estabelecidos na literatura (HAIR et al, 2014).

Tabela 5 Descrição das variáveis da Utilidade Percebida

\begin{tabular}{lcccc}
\hline Variáveis & / & \multicolumn{3}{c}{ Composite } \\
Critérios & AVE & Alfa de Cronbach & reliability & Rho-A \\
QUALIINFO & 0,62 & 0,90 & 0,92 & 0,90 \\
QUALISERV & 0,59 & 0,88 & 0,91 & 0,89 \\
QUALISIS & 0,72 & 0,92 & 0,94 & 0,93 \\
SATISF & 1,00 & 1,00 & 1,00 & 1,00 \\
UTIPER & 0,76 & 0,95 & 0,96 & 0,95 \\
Limite & 0,50 & 0,70 & 0,70 & 0,70 \\
\hline
\end{tabular}

Fonte: Elaboração Própria (2017)

A AVE é a porção dos dados (nas respectivas variáveis) que é explicada por cada um dos constructos, respectivos aos seus conjuntos de variáveis ou quanto, em média, as variáveis se correlacionam positivamente com os seus respectivos constructos. Assim, quando as AVE são maiores que 0,50 admite-se que o modelo converge a um resultado satisfatório. (FORNELL e LARCKER, 1981; HAIR et al., 2014)

A tabela 6 apresenta os resultados da valide discriminante observando os critérios de Fornell-Larcker, que formam uma abordagem mais conservadora para avaliar a validade discriminante dos itens. Os critérios comparam a raiz quadrada dos valores de AVE com as 
correlações entre os construtos. A raiz quadrada da AVE de cada construto deve exceder o maior valor das correlações dos demais construtos. O racional para esse método fundamentase na concepção de que um construto compartilha mais variância com seus indicadores associados do que com qualquer outro construto. Os resultados encontrados evidenciam que os critérios foram respeitados para todas as variáveis analisadas. O mesmo ocorreu para a análise das cargas cruzadas dos indicadores que se mantiveram superiores à carga cruzadas dos outros constructos.

Tabela 6 Validade Discriminante

\begin{tabular}{llclcl}
\hline & QUALIINFO & QUALISERV & QUALISIS & SATISF & UTIPER \\
\hline QUALIINFO & 0,79 & & & & \\
QUALISERV & 0,75 & 0,77 & & & \\
QUALISIS & 0,78 & 0,79 & 0,85 & & \\
SATISF & 0,71 & 0,73 & 0,77 & 1,00 & \\
UTIPER & 0,77 & 0,77 & 0,82 & 0,84 & 0,87
\end{tabular}

Fonte: Elaboração Própria (2017)

\subsubsection{Resultados do Modelo Estrutural}

O modelo estrutural foi avaliado considerado a colinearidade, significância e relevância dos relacionamentos do modelo estrutural, avaliação da qualidade e capacidade preditiva. A análise da colinearidade por meio do fator de variância (VIF) obteve valores considerados satisfatórios indicando que todas as variáveis podem permanecer no modelo (HAIR et al., 2014). Como apresentado na Tabela 7, todos os valores são adequados.

Tabela 7 Resultados de VIF obtidos

\begin{tabular}{lc}
\hline Critério & SATISF \\
QUALIINFO & 3,12 \\
QUALISERV & 3,29 \\
QUALISIS & 4,10 \\
SATISF & \\
UTIPER & 3,85 \\
\hline Limite & 5,00 \\
\hline
\end{tabular}

Fonte: Elaboração Própria (2017) 
Mediante a realização do procedimento de bootstrapping, foi possível encontrar os resultados para os coeficientes de caminho e valores da estatística $\mathrm{t}$ de student e p-value, conforme Tabela 8 . Com os valores apresentados, verificou-se com o nível de $95 \%$ que todos os caminhos foram confirmados de acordo com as hipóteses com sinais positivos indicando a relação de influência das variáveis para a satisfação dos usuários. A significância estatística das variáveis latentes pode ser comprovada pelo valor do p-value. Assim, todas as relações entre as variáveis latentes foram consideradas consistentes, sendo que a utilidade percebida é o constructo que apresenta o maior valor beta do caminho.

Tabela 8 Significância estatística dos coeficientes de caminho

\begin{tabular}{llllll}
\hline Relações & $\begin{array}{l}\text { Beta } \\
\text { caminho }\end{array}$ & $\begin{array}{c}\text { do } \\
\text { Valor } \\
\text { Médio }\end{array}$ & $\begin{array}{l}\text { Desvio } \\
\text { Padrão }\end{array}$ & Estatística t & P Value \\
\hline QUALIINFO -> SATISF & 0,06 & 0,06 & 0,03 & 2,36 & 0,02 \\
QUALISERV -> SATISF & 0,11 & 0,11 & 0,03 & 4,04 & 0,00 \\
QUALISIS -> SATISF & 0,16 & 0,16 & 0,03 & 5,11 & 0,00 \\
UTIPER -> SATISF & 0,58 & 0,58 & 0,03 & 20,20 & 0,00 \\
\hline
\end{tabular}

Fonte: Elaboração Própria (2017)

O modelo apresenta $\mathrm{R}^{2}$ e $\mathrm{R}^{2}$ ajustado de $73,33 \%$, considerado adequado e com efeito grande (COHEN,1988). Outros testes importantes que foram realizados são a Relevância Preditiva $\left(\mathrm{Q}^{2}\right)$ ou indicador de Stone-Geisser e o Tamanho do Efeito $\left(\mathrm{f}^{2}\right)$ ou indicador de Cohen. O primeiro teste avalia a precisão (ou acurácia) do modelo ajustado. O critério de avaliação são valores maiores que zero (HAIR et al., 2014). Já no segundo teste o valor é obtido pela inclusão e exclusão de constructos do modelo (um a um). Avalia-se quanto cada constructo é "útil" para o ajuste do modelo. Valores próximos a 0,02, 0,15 e 0,35 são considerados pequenos, médios e grandes, respectivamente (HAIR et al., 2014; OLIVEIRA, 2014). A tabela 9 apresenta os resultados encontrados.

Tabela 9 Redundância e comunalidade dos constructos

\begin{tabular}{lll}
\hline Relações & $\mathbf{Q}^{\mathbf{2}}$ & $\mathbf{f}^{\mathbf{2}}$ \\
\hline QUALIINFO -> SATISF & 0,48 & 0,00 \\
QUALISERV -> SATISF & 0,44 & 0,01 \\
QUALISIS -> SATISF & 0,58 & 0,02 \\
UTIPER -> SATISF & 1,00 & 0,32 \\
\hline
\end{tabular}

Fonte: Elaboração Própria (2017) 


\subsection{DISCUSSÃO DOS RESULTADOS}

Por meio da análise do modelo apresentado na figura 2, verificou-se que a utilidade percebida teve maior impacto na determinação da satisfação dos usuários, seguida pela Qualidade do Sistema, Qualidade do Serviço e Qualidade da Informação. O artigo confirmou todas as hipóteses propostas. Assim, a "H1 - A qualidade do sistema influencia positivamente o nível de satisfação do usuário" foi aceita, sendo que o valor beta encontrado para a relação entre a variável QUALISIS->SATISF foi 0,164. O modelo final, com os indicadores, as variáveis latentes e informando os valores de beta para as relações causais entre essas variáveis para a determinação da satisfação são apresentados na figura 2 a seguir.

Figura 2 Modelo Final com as cargas fatoriais e valor beta do coeficiente de caminho

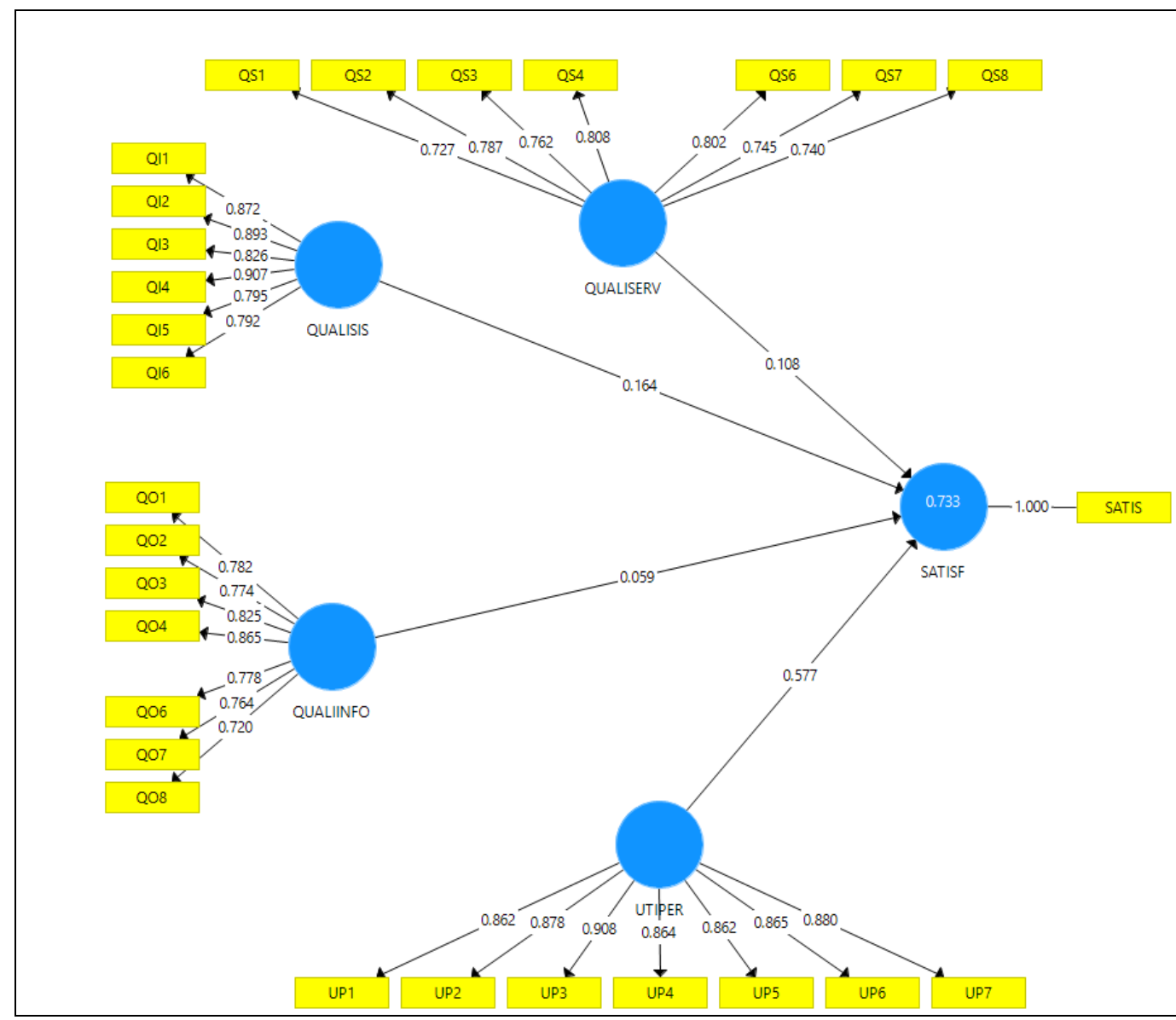

Fonte: Adaptado do modelo de Ainin, Bahri e Admad (2012)

A hipótese "H2 - A qualidade da informação influencia positivamente o nível de satisfação do usuário" também foi aceita, medida pela relação entre QUALIINFO->SATISF apresentada na figura com valor beta 0,059. A hipótese "H3 - A qualidade do serviço 
influencia positivamente o nível de satisfação do usuário" também foi confirmada (QUALISERV->SATISF com valor beta 0,108). A hipótese "H4 - A utilidade percebida influencia positivamente o nível de satisfação do usuário", sendo que a relação UTIPER$>$ SATISF apresentou o maior valor beta.

\section{CONSIDERAÇÕES FINAIS}

Apesar de apresentar poucas contribuições teóricas para o estudo da satisfação dos usuários dos sistemas acadêmico, esse estudo apresenta resultados empíricos importantes. Teoricamente foi apresentada a utilização de um modelo de avaliação da satisfação e do ponto de vista prático, esse estudo possibilitou que a IES analisada conhecesse os fatores determinantes da satisfação dos usuários de um sistema de informação acadêmico.

Os resultados encontrados evidenciaram que o fator utilidade percebida, que considera variáveis como facilidade de acessar o sistema, layout e quantidade de informações exibidas na tela, teve maior impacto na determinação da satisfação dos usuários, seguido pelos fatores Qualidade do Sistema, Qualidade do Serviço e Qualidade da Informação. Dessa forma, podese entender que o sistema de gestão acadêmico avaliado tem fornecido informações consideradas de qualidade pelos usuários. Os resultados encontrados possibilitarão que a IES desenvolva ações para melhorar continuamente o sistema de como a aumentar ainda mais a satisfação dos usuários.

Do ponto de vista estatístico, o estudo atendeu todos os critérios para validação do modelo, além de apresentar um bom nível de ajuste, mostrando-se estatisticamente adequado. Todas as hipóteses de pesquisa foram aceitas. Percebe-se, assim, que a utilização do modelo de Ainin, Bahri e Admad (2012) é apropriado para explicar a estrutura relacional dos dados, apesar de não afirmar que esse modelo é único, demonstra que o referencial teórico considerado adequado para os dados em observação, não excluindo, naturalmente, outros modelos teóricos que poderiam ter sido utilizados.

Sugere-se para pesquisas futuras que o mesmo modelo seja aplicado aos outros usuários do sistema acadêmico possibilitando comparar os resultados encontrados e em outras Universidades, a fim de verificar a divergência ou convergência dos resultados já obtidos. De forma semelhante, acrescentar ou modificar variáveis trará novos resultados que poderão adicionar conhecimento ao tema. 


\section{REFERÊNCIAS}

AININ, S.; BAHRI, S.; AHMAD, A. Evaluating portal performance - A study of the National Higher Education Fund (PTPTN) Corporation portal. Telematics and Informatics, v. 29, n. 3, p. 314-323, 2012.

BARROCA FILHO, I. M; AQUINO JÚNIOR, G. S.A case study of development of a mobile application from an existing web information system. Int J Of Web Info Systems, v. 12, n. 1, p.18-38, 18 abr. 2016.

BOURNARIS, T., MANOS, B., MOULOGIANNI, C., KIOMOURTZI, F., TANDINI, M.; Measuring Users Satisfaction of an e-Government portal. Procedia Technology . v.8, 371$377,2013$.

CARVALHO, S. R.; MELO FILHO, I. J. de; VIDAL, T. C.; MELO, R. M. de; GOMES, A. S. Integração entre o sistema de gestão da aprendizagem: identificando necessidades e prototipando requisitos favoráveis a prática docente. Revista Brasileira de Computação Aplicada, v. 4, n. 1, p. 81-91, mar. 2012.

CLAYTON, P. D.; CROSSWELL, A. Network Information Security In A Phase Hi. Integrated Academic Information Management System (Iaims). 1993.

DELONE, W. H.; MCLEAN, E. R.., The DeLone and McLean model of information systems success: A ten-year update. Journal of Management Information Systems, v.19, n 4, 9-30, 2003.

Information Systems Success: The Quest for the Dependent Variable. Information Systems Research.v.3, n.1, 60-95. 1992.

DOLL, W., \& TORKZADEH, G. The measurement of end-user computing satisfaction. Mis Quarterly. v.12, n.2, 259-274. 1988.

DONG, T., CHENG, N., WU, Y. J.; A study of the social networking website service in digital content industries: The Facebook case in Taiwan. Computers in Human Behavior, v. 30, 708-714, 2014.

HAIR, J.; BABIN, B.; MONEY, A.; SAMUEL, P. Fundamentos de Métodos de Pesquisa em Administração. Porto Alegre: Bookman, 2005.

HAIR, J.F., RINGLE, C.M., SARSTEDT, M.: Partial Least Squares: The Better Approach to Structural Equation Modeling? Long Range Planning, Volume 45 (2012), Issue 5-6, pp. $312-$ 319.

HAIR, J. F. et al. Partial least squares structural equation modeling (PLS-SEM). [s.1: s.n.]. v. 26, 2014. 
LEE, H. S.; CHOI, Y. H.; JO, N. O. Determinants Affecting User satisfaction with campus Portal Services in Korea. Journal of internet Banking \& Commerce, v. 14, n. 1, 2009.

LIMA, L. F. F. M. Percepção de segurança em sistemas de informação e sua relação com a qualidade percebida de serviços, perfil de liderança e perfil dos seguidores, entre as diretorias do Inmetro. 2006. Dissertação (Mestrado em Sistemas de Gestão) - Universidade Federal Fluminense, Niterói, 2006.

MATTOS, J. R. S. ERP: a hora do retorno. Informationweek, p. 26-27, Nov 1999.

NUNNALLY, J. C. Psychometric theory. [s.1.] McGraw-Hill, 1994.

OLIVER, R. L.; A cognitive model of the antecedents and consequences of satisfaction decisions. Journal of Marketing Research, v.17, n4, 460-469, 1980.

QUEIROZ, F. C. B. P.; HÉKIS, H. R.; QUEIROZ, J. V.; OLIVEIRA, L. A. B.; VASCONCELOS, N. V. C. de. Contribuição dos Sistemas Integrados de gestão para as práticas de ensino e aprendizagem. Augusto Guzzo Revista Acadêmica, v. 1, n. 9, p.45-52, 2012.

REIS, P. N. C.; PITASSI, C.; BOUZADA, M. A. C. Os fatores que explicam o grau de aceitação de um sistema de informação acadêmica utilizado nos processos de apoio à gestão docente: um estudo de caso em uma IES privada. In: Simpósio de Administração da Produção, Logística e Operações Internacionais, XV, 2012, Anais ... São Paulo, 2012.

RIGDON, E. E. Choosing PLS path modeling as analytical method in European management research: A realist perspective. European Management Journal, v. 34, n. 6, p. 598-605, dez. 2016.

RINGLE, C. M., WENDE, S., AND BECKER, J.-M.. "SmartPLS 3." Boenningstedt: SmartPLS GmbH, http://www.smartpls.com, 2015

SANTOS, M. dos. Perfis de gerenciamento estratégico a informação nas empresas brasileiras. Brazilian Business Review, v. 3, n. 2, p. 118-136, jan./jun.2006.

SENGER I.; BRITO, M. J. Gestão de sistema de informação Acadêmica: Um Estudo Descritivo da Satisfação dos Usuários. Revista de Administração Mackenzie, v. 6, n.3, p. 12-40, 2005.

SARSTEDT, M. et al. Estimation issues with PLS and CBSEM: Where the bias lies! Journal of Business Research, v. 69, n. 10, p. 3998-4010, out. 2016.

SILVA, C. A. B. da. Arquitetura empresarial: um estudo de caso sobre a integração entre a plataforma Moodle e a SIGAA na UFRN. 2012. 141 f. Dissertação (Mestrado em Administração) Universidade Federal do Rio Grande do Norte, Natal, 2012. 